\title{
FIVE-DIMENSIONAL RELATIVITY AND TWO TIMES
}

Paul S. Wesson ${ }^{1,2}$

1. Dept. Physics, University of Waterloo, Waterloo, Ontario N2L 3G1, Canada

Keywords: Time, 5D Relativity, Waves, Equivalence Principle

Pacs: 05.70.Ln, 04.50.+h, 0.4.20.Cv

2. Correspondence: mail $=(1)$ above; fax $=(519) 746-8115$;

email=wesson@astro.uwaterloo.ca. 


\section{FIVE-DIMENSIONAL RELATIVITY AND TWO TIMES}

\section{$\underline{\text { Abstract }}$}

It is possible that null paths in 5D appear as the timelike paths of massive particles in $4 \mathrm{D}$, where there is an oscillation in the fifth dimension around the hypersurface we call spacetime. A particle in 5D may be regarded as multiply imaged in $4 \mathrm{D}$, and the $4 \mathrm{D}$ weak equivalence principle may be regarded as a symmetry of the 5D metric.

\section{Introduction}

In quantum theory, it has recently been shown that the statistical interactions of particles can lead to thermodynamic arrows of time for different parts of the universe which are different or even opposed [1]. In relativity

theory, it is well known how to incorporate the phenomenological laws of standard thermodynamics into general relativity, but it has not been clear how to treat particle dynamics and the nature of time in higher-dimensional manifolds which may unify quantum theory and gravity. However, a couple of exact solutions of the field equations of 5D relativity have recently 
been found which have good physical properties but involve manifolds with signature $[+(---)+]$ that describe two "time" dimensions [2]. Such twotime metrics are currently the subject of investigation in relation to even higher-dimensional extensions of general relativity, notably string theory [3]. While field theory in $N$ dimensions is an alluring subject, concrete calculations which might be compared to observations are unfortunately compromised somewhat by uncertainty about $N(4<N \leq 26$ is a commonly-held view). The case $N=5$ continues to be a major focus, because it is the basic extension of general relativity and the low-energy limit of more extended theories. Thus $N=5$ induced-matter theory and membrane theory are widely regarded as the best options for resolving the cosmological-constant and hierarchy problems for the energy density of the vacuum and masses of particles, because they both drop the hobbling cylinder condition (no dependence on the extra coordinate) typical of old Kaluza-Klein theory. The versatility of $5 \mathrm{D}$ relativity has recently been illustrated again by the demonstration that timelike paths in $4 \mathrm{D}$ can be interpreted as null paths in $5 \mathrm{D}[4,5]$. This implies that what we regard as massive particles moving through spacetime, with finite separations in ordinary 3D space and 4D proper time, are photonlike objects in 5D with no "separation" in supertime. Such an interpretation 
should not be viewed as merely a neat concept, because if we use the $4 \mathrm{D}$ proper time of conventional relativity to parametize the motion of a particle in $5 \mathrm{D}$, the geodesic equations for the latter show that in general there is a fifth force which acts parallel to the 4-velocity in both induced-matter and membrane theory and is in principle open to observation $[6,7]$. The implications of this are major, irrespective of whether we live in a world where matter is the manifestation of an unconstrained fifth dimension $[2,4,6,8]$ or in one where we are constrained to a hypersurface in a shadowy five-dimensional "bulk" $[3,5,7,9]$. The two approaches appear to be equivalent [10], at least mathematically.

In what follows we wish to extend what has been noted above and derive several new results in two-time 5D relativity that are remarkable.

\section{Properties of Null 5D Two-Time Metrics}

By Campbell's theorem, it is always possible to embed a 4D Riemannian line element with $d s^{2}=g_{\alpha \beta} d x^{\alpha} d x^{\beta}$ in a 5D one with $d S^{2}=g_{A B} d x^{A} d x^{B}$ (here $g_{\alpha \beta}$ and $g_{A B}$ are the $4 \mathrm{D}$ and $5 \mathrm{D}$ metric tensors with $\alpha=0,123$ and $A=$ $0,123,4)$. However, $g_{A B}=g_{A B}\left(x^{\alpha}, l\right)$ where $x^{4}=l$ is the extra coordinate, 
so $5 \mathrm{D}$ quantities calculated from $g_{A B}$ will in general be $Q=Q\left(x^{\alpha}, l\right)$. The latter behave covariantly under the group of 5D coordinate transformations $x^{A} \rightarrow \bar{x}^{A}\left(x^{B}\right)$, but $\underline{\text { not }}$ under the conventional group of 4D transformations $x^{\alpha} \rightarrow \bar{x}^{\alpha}\left(x^{\beta}\right)$. Thus a choice of $5 \mathrm{D}$ coordinates (or gauge) is necessary in order to specify $4 \mathrm{D}$ physics.

In the quasi-Minkowski gauge, a particle moving along a null path in a two-time 5D metric has

$$
0=d S^{2}=d t^{2}-\left(d x^{2}+d y^{2}+d z^{2}\right)+d l^{2}
$$

The 5-velocities $U^{A} \equiv d x^{A} / d \lambda$ where $\lambda$ is an affine parameter obey $U^{A} U_{A}=$ 0 (we absorb the speed of light $c$ here, and the gravitational constant $G$ and Planck's constant $h$ elsewhere, by a choice of units that renders them all unity). With $\lambda=s$ for the proper $4 \mathrm{D}$ time, the velocity in ordinary space $(v)$ is related to the velocity along the axis of ordinary time $(u)$ and the velocity along the fifth dimension $(w)$ by $v^{2}=u^{2}+w^{2}$. This implies super-luminal speeds; but the particle which follows the path specified by (1) should not be identified with the tachyon of special relativity, because in both inducedmatter $[2,4]$ and brane theory $[5,7] l$ is related to the rest mass of a test particle. 
In the non-electromagnetic gauge, the line element can be written

$$
d S^{2}=g_{\alpha \beta}\left(x^{\gamma}, l\right) d x^{\alpha} d x^{\beta}+\epsilon \Phi^{2}\left(x^{\gamma}, l\right)
$$

This uses only 4 of the 5 available degrees of coordinate freedom to remove the electromagnetic potentials $\left(g_{4 \alpha}\right)$, leaving the scalar or Higgs potential and the signature general $\left(g_{44}=\epsilon \Phi^{2}\right)$. The gravitational potentials $\left(g_{\alpha \beta}\right)$ are also general. The components of the 5D Ricci tensor for the metric (2) deserve to be generally known, and we therefore tabulate them in what we believe to be their most convenient form:

$$
\begin{aligned}
&{ }^{5} R_{\alpha \beta}={ }^{4} R_{\alpha \beta}-\frac{\Phi, \alpha ; \beta}{\Phi}+\frac{\epsilon}{2 \Phi^{2}}\left(\frac{\Phi_{, 4} g_{\alpha \beta, 4}}{\Phi}-g_{\alpha \beta, 44}\right. \\
&\left.+g^{\lambda \mu} g_{\alpha \lambda, 4} g_{\beta \mu, 4}-\frac{g^{\mu \nu} g_{\mu \nu, 4} g_{\alpha \beta, 4}}{2}\right) \\
& R_{44}=-\epsilon \Phi \square \Phi-\frac{g^{\lambda \beta}{ }_{, 4} g_{\lambda \beta, 4}}{2}-\frac{g^{\lambda \beta} g_{\lambda \beta, 44}}{2}+\frac{\Phi_{, 4} g^{\lambda \beta} g_{\lambda \beta, 4}}{2 \Phi} \\
&-\frac{g^{\mu \beta} g^{\lambda \sigma} g_{\lambda \beta, 4} g_{\mu \sigma, 4}}{4} \\
& R_{4 \alpha}=\quad \Gamma\left(\frac{g^{\beta \lambda} g_{\lambda \alpha, 4}-\delta_{\alpha}^{\beta} g^{\mu \nu} g_{\mu \nu, 4}}{2 \Gamma}\right)_{, \beta} \\
&+ \frac{g^{\mu \beta} g_{\mu \beta, \lambda} g^{\lambda \sigma} g_{\sigma \alpha, 4}}{4}-\frac{g^{\lambda \beta} g_{\beta \mu, \alpha} g^{\mu \sigma} g_{\sigma \lambda, 4}}{4} .
\end{aligned}
$$

Here a comma denotes the ordinary partial derivative, a semicolon denotes the ordinary $4 \mathrm{D}$ covariant derivative, $\square \Phi \equiv g^{\mu \nu} \Phi_{, \mu ; \nu}$ and $\Gamma \equiv\left|\epsilon \Phi^{2}\right|^{1 / 2}$. The 
field equations of $5 \mathrm{D}$ relativity are commonly taken to be $R_{A B}=0$. Then (4) is a wave equation for the scalar field, (5) can be couched as a set of 4 conservation equations, and (3) can be put into the form of the 10 Einstein equations $[2,4,10]$. The latter $\operatorname{read} G_{\alpha \beta}=8 \pi T_{\alpha \beta}$, where the Einstein tensor $G_{\alpha \beta} \equiv{ }^{4} R_{\alpha \beta}-{ }^{4} R g_{\alpha \beta} / 2$ is constructed from the remaining $5 \mathrm{D}$ quantities. Using $R_{A B}=0$ in $(3)-(5)$, the $4 \mathrm{D}$ scalar curvature is

$$
{ }^{4} R=\frac{\epsilon}{4 \Phi^{2}}\left[g_{, 4}^{\mu \nu} g_{\mu \nu, 4}+\left(g^{\mu \nu} g_{\mu \nu, 4}\right)^{2}\right]
$$

This relation has been used implicitly in the literature, but explicitly as here it is extremely instructive: (a) what we call the curvature of $4 \mathrm{D}$ spacetime can be regarded as the result of embedding it in an $x^{4}$-dependent 5D manifold; (b) the sign of the 4D curvature depends on the signature of the 5D metric, which here is $+(---) \pm$ and admits the two-time option; (c) the magnitude of the 4D curvature depends strongly on the scalar field or the size of the extra dimension $\left(g_{44}=\epsilon \Phi^{2}\right)$, so while it may be justifiable to neglect this in astrophysics (where the $4 \mathrm{D}$ curvature is small) it can be crucial in cosmology and particle physics. The 4D energy-momentum tensor that follows from 
$(3)-(5)$ is

$$
\begin{aligned}
8 \pi T_{\alpha \beta}= & \frac{\Phi_{, \alpha ; \beta}}{\Phi}-\frac{\epsilon}{2 \Phi^{2}}\left\{\frac{\Phi_{, 4} g_{\alpha \beta, 4}}{\Phi}-g_{\alpha \beta, 44}+g^{\lambda \mu} g_{\alpha \lambda, 4} g_{\beta \mu, 4}\right. \\
& \left.-\frac{g^{\mu \nu} g_{\mu \nu, 4} g_{\alpha \beta, 4}}{2}+\frac{g_{\alpha \beta}}{4}\left[g^{\mu \nu}{ }_{, 4} g_{\mu \nu, 4}+\left(g^{\mu \nu} g_{\mu \nu, 4}\right)^{2}\right]\right\} .
\end{aligned}
$$

The existence of this relation has been inferred in the literature as a corollary of Campbell's theorem, but explicitly as here it is also extremely instructive: (a) what we call matter in a curved 4D spacetime can be regarded as the result of the embedding in an $x^{4}$-dependent (possibly flat) 5D manifold; (b) the nature of the 4D matter depends on the signature of the 5D metric; (c) the 4D source depends on the extrinsic curvature of the embedded 4D spacetime and the scalar field associated with the extra dimension, which while they are in general mixed correspond loosely to ordinary matter and the stress-energy of the vacuum.

In the pure-canomical gauge of induced-matter theory, the first part of (2) is factorized via $g_{\alpha \beta}\left(x^{\gamma}, l\right)=\left(l^{2} / L^{2}\right) \bar{g}_{\alpha \beta}\left(x^{\gamma}\right)$ where $L$ is a constant length, and the last part of $(2)$ is fixed via $g_{44}=-1$. There is a considerable literature on this gauge $[2,4]$, which is related to the warp gauge of membrane theory $[5,7$ : the latter uses a factor that is exponential in $l]$. Then (7) causes Einstein's equations to read $G_{\alpha \beta}=-3 \epsilon \bar{g}_{\alpha \beta} / L^{2}$, which defines a cosmological constant $\Lambda=-3 \epsilon / L^{2}$. [Relation (6) also gives the standard relation for an 
embedded vacuum spacetime.] The cosmological constant can, of course, be regarded as defining the energy density and pressure of the vacuum in Einstein's theory via $\rho=-p=\Lambda / 8 \pi$. This is a special case of (7), and we now turn our attention to the general relations (3)-(7) with an extra timelike dimension.

A simple, wave-like solution of $R_{A B}=0$ is given by

$$
d S^{2}=\frac{l^{2}}{L^{2}}\left[d t^{2}-e^{i\left(\omega t+k_{x} x\right)} d x^{2}-e^{i\left(\omega t+k_{y} y\right)} d y^{2}-e^{i\left(\omega t+k_{z} z\right)} d z^{2}\right]+d l^{2} .
$$

Here $k_{x y z}$ are wave numbers and the frequency is constrained by the solution to be $\omega= \pm 2 / L$. We have studied (8) both algebraically and computationally using the program GRTensor (which may also be used to verify it). Solution (8) clearly has two "times". It also has complex metric coefficients for the ordinary 3D space, but closer inspection shows that the structure of the field equations leads to physical quantities that are real. The $3 \mathrm{D}$ wave is not of the sort found in general relativity, but owes its existence to the choice of coordinates. A trivial change in the latter suppresses the appearance of the wave in 3D space, in analogy to how a transverse wave is noticed or not by an observer, depending on whether he is fixed in the laboratory frame or moving with the wave. A further change of coordinates can be shown to make (8) look like the 5D analog of the de Sitter solution. This leads us to conjecture 
that the wave is supported by the pressure and energy density of a vacuum with the equation of state found in general relativity, namely $p+\rho=0$. This is confirmed to be the case, with $\Lambda<0$. It may also be confirmed that (8) is not only Ricci-flat $\left(R_{A B}=0\right)$ but also Riemann-flat $\left(R_{A B C D}=0\right)$. It is a wave travelling in a curved $4 \mathrm{D}$ spacetime that is embedded in a flat $5 \mathrm{D}$ manifold which has no energy.

The logical condition on the path, for a particle moving in a 5D manifold that has no energy, is that it be null. To make contact with other work we choose a two-time metric of canonical form, so

$$
0=d S^{2}=\frac{l^{2}}{L^{2}} d s^{2}+d l^{2}
$$

Here we take $d s^{2}=\bar{g}_{\alpha \beta}\left(x^{\alpha}, l\right) d x^{\alpha} d x^{\beta}$, using all of the 5 available coordinate degrees of freedom to suppress the potentials of electromagnetic and scalar type, but leaving the metric otherwise general. The solution of (9) is $l=l_{0} \exp \left[ \pm i\left(s-s_{0}\right) / L\right]$, where $l_{0}$ and $s_{0}$ are constants of which the latter may be absorbed. Then $l=l_{0} e^{ \pm i s / L}$ describes an $l$-orbit that oscillates about spacetime with amplitude $l_{0}$ and wavelength $L$. The motion is actually simple harmonic, since $d^{2} l / d s^{2}=-l / L^{2}$. Also, $d l / d s= \pm i l / L$, so the physical identifications of the mass of a particle with the momentum in the extra dimension as in brane theory $[5,7]$ or with the extra coordinate as 
in induced-matter theory $[2,4]$ are equivalent, modulo a constant. In both cases, the $l$-orbit intersects the $s$-plane an infinite number of times. There is only one period in the metric (9), defined by $L$, but of course a Fourier sum of simple harmonics can be used to construct more complicated orbits in the $l / s$ plane. [Alternatively, extra length scales can be introduced to (9) via $L^{2} \rightarrow\left(L_{1}^{2} L_{2}^{2} L_{3}^{2} \ldots L_{n}^{2}\right) /\left(L_{2}^{2} L_{3}^{2} \ldots L_{n}^{2}+L_{1}^{2} L_{3}^{2} \ldots L_{n}^{2}+\ldots\right)$.] If we identify the orbit in the $l / s$ plane with that of a particle, we have a realization of the old idea (often attributed to Wheeler and/or Feynmann) that instead of there being $10^{80}$ particles in the visible universe there is in fact only one which appears $10^{80}$ times.

The above description is classical, but there is no impediment to its extension to the quantum domain [2]. By (9), the traditional sum over $s$-paths in $4 \mathrm{D}$ can if so desired be replaced by a sum over $l$-paths in $5 \mathrm{D}$. However, metrics like (9) still require that the 5D path $S$ be minimized around zero. The condition for this is given by the 5D geodesic equation, which for (9) can conveniently be presented in terms of the equation of motion in spacetime 
and the equation of motion in the extra dimension:

$$
\begin{aligned}
\frac{d u^{\mu}}{d s}+\Gamma_{\beta \gamma}^{\mu} u^{\beta} u^{\gamma} & =f^{\mu} \\
f^{\mu} & \equiv\left(-g^{\mu \alpha}+\frac{u^{\mu} u^{\alpha}}{2}\right) u^{\beta} \frac{d l}{d s} \frac{\partial g_{\alpha \beta}}{\partial l} \\
\frac{d^{2} l}{d s^{2}}-\frac{2}{l}\left(\frac{d l}{d s}\right)^{2}-\frac{l}{L^{2}} & =\frac{1}{2}\left\{\frac{l^{2}}{L^{2}}+\left(\frac{d l}{d s}\right)^{2}\right\} u^{\alpha} u^{\beta} \frac{\partial g_{\alpha \beta}}{\partial l} .
\end{aligned}
$$

Here $u^{\mu} \equiv d x^{\mu} / d s, \Gamma_{\beta \gamma}^{\mu}$ is the usual $4 \mathrm{D}$ Christoffel symbol, and $f^{\mu}$ is the fifth force (per unit inertial mass) which has been discussed for induced-matter theory [6] and membrane theory [7]. By (10), the motion in spacetime is only geodesic in the usual $4 \mathrm{D}$ sense if $f^{\mu}=0$. This force may be split into a part $\left(-g^{\mu \alpha}+u^{\mu} u^{\alpha}\right) u^{\beta}(d l / d s)\left(\partial g_{\alpha \beta} / \partial l\right)$ which by construction is normal to the 4-velocity $u^{\mu}$, and a part - $\left(u^{\mu} / 2\right)\left(u^{\alpha} u^{\beta} \partial g_{\alpha \beta} / \partial l\right)(d l / d s)$ which is parallel to it. The latter has no analog in 4D general relativity or any field theory like it, including electromagnetism. The question of why the fifth force has hitherto not been observed is therefore tantamount to the question of why the scalar quantity $Q \equiv u^{\alpha} u^{\beta}\left(\partial g_{\alpha \beta} / \partial l\right)$ is small. [Clearly $u^{\mu} \neq 0$ in general, $d l / d s=0$ would effectively reduce the metric from $5 \mathrm{D}$ to $4 \mathrm{D}$, and the null wave $l=l_{0} e^{ \pm i s / L}$ satisfies (12) with no constraint on $Q$.] Our answer to this is as follows: $Q=0$ if $\partial g_{\alpha \beta} / \partial l=0$, meaning that there is no intrusion of the fifth dimension into spacetime, irrespective of the physical identification 
of $x^{4}=l$. This says that the $4 \mathrm{D}$ weak equivalence principle is a symmetry of the 5D metric.

\section{Conclusion}

There are non-unique times in 4D statistical mechanics [1], 5D relativity [2] and $N(>5) D$ string theory [3]. In 5D, particles which are dynamically massless can appear to be massive in spacetime $[4,5]$, and are acted upon in general by a fifth force $[6,7]$ which can manifest itself in both induced-matter and membrane theory $[8,9]$, though these approaches are mathematically equivalent [10]. In the present work, we have outlined the main consequences of $5 \mathrm{D}$ relativity with signature $[+(---)+]$. Though technically referred to as "two-time" metrics, there is no problem with closed timelike paths because the second timelike coordinate is related to the (inertial) rest mass of a particle in both induced-matter and membrane theory $[2,7]$. To derive physical results requires the specification of a gauge, as in (1) or (2). However, we have given the components of the $5 \mathrm{D}$ Ricci tensor $R_{A B}$ for a general non-electromagnetic gauge in (3)-(5). These for field equations $R_{A B}=0$

lead to expressions for the embedded 4D Einstein space, namely (6) for the 
curvature scalar and (7) for the energy-momentum tensor. The exact solution (8) illustrates the new physics deriveable from two-time metrics: it describes a wave moving through a de Sitter vacuum. The general two-time metrics (9) describe waves in the fifth dimension that oscillate around the hypersurface we call spacetime. This can be used as a model for multiply-imaged particles. The dynamics of the latter are governed by relations (10)-(12), which show that the fifth force typical of induced-matter and membrane theory is absent if the weak equivalence principle is invoked as a symmetry of the metric.

\section{Acknowledgements}

This work grew out of earlier collaborations with H. Liu, B. Mashhoon and S.S. Seahra. It was supported by N.S.E.R.C.

\section{$\underline{\text { References }}$}

1. L.S. Schulman, Phys. Rev. D $\underline{7}, 2868$ (1973). L.S. Schulman, Time's Arrow and Quantum Measurement (Cambridge Un. Press, Cambridge, 1997). L.S. Schulman, Phys. Rev. Lett. $\underline{83}, 5419$ (1999). L.S. Schulman, Phys. Rev. Lett. $\underline{85}, 897$ (2000).

2. P.S. Wesson, Space-Time-Matter (World Scientific, Singapore, 1999). A. Billiard, P.S. Wesson, Phys. Rev. D $\underline{53}, 731$ (1996).A. Billiard, P.S. 
Wesson, Gen. Rel. Grav. 228, 129 (1996). P.S. Wesson, Observatory 121, 82 (2001). P.S. Wesson, J. Math. Phys. in press (2002). (grgc/0105059, 2001.)

3. I. Bars, C. Kounnas, Phys. Rev. D $\underline{56}, 3664$ (1997). I. Bars, C. Deliduman, O. Andreev, Phys. Rev. D $\underline{58}, 066004$ (1998). I. Bars, C. Deliduman, D. Minic, Phys. Rev. D $\underline{59}, 125004$ (1999). J. Kocinski, M. Wierzbicki, gr-gc/0110075 (2001).

4. S.S. Seahra, P.S. Wesson, Gen. Rel. Grav. 3ㅜ 1731 (2001).

5. D. Youm, hep-th/0110013 (2001).

6. P.S. Wesson, B. Mashhoon, H. Liu, W.N. Sajko, Phys. Lett. B $\underline{456}, 34$ (1999).

7. D. Youm, Phys. Rev. D $\underline{62}, 084002$ (2000).

8. P.S. Wesson, Phys. Lett. B $\underline{276}, 299$ (1992).

9. L. Randall, R. Sundrum, Mod. Phys. Lett. A $\underline{13}, 2807$ (1998).

10. J. Ponce de Leon, Mod. Phys. Lett. A $\underline{16}, 2291$ (2001). 